Publ. RIMS, Kyoto Univ.

12 Suppl. (1977), 343-346.

\title{
Complex-dimensional Integral and Light-cone Singularities
}

\author{
by \\ Noboru NAKANISHI*
}

\begin{abstract}
The notion of a complex-dimensional integral is introduced in the complex $n$-dimensional Minkowski space. Its basic properties, such as Lorentz invariance, are investigated. Complex-dimensional invariant delta functions $\Delta_{n}\left(x ; m^{2}\right), \Delta^{(1)}{ }_{n}\left(x ; m^{2}\right)$, etc. are explicitly calculated in position space. It is proposed to define products of singular functions in the ordinary Minkowski space by analytically continuing the corresponding $n$-dimensional ones to $n=4$. The light-cone singularities of $\left[\Delta\left(x ; m^{2}\right)\right]^{2}, \Delta\left(x ; m^{2}\right)$ $\times \Delta^{(1)}\left(x ; m^{2}\right)$ and $\left[\Delta^{(1)}\left(x ; m^{2}\right)\right]^{2}$ are shown to be unambiguously determined in this way.
\end{abstract}

Recently, in quantum field theory, much attention has been paid to complex-dimensional regularization [1]. The momentum-space Feynman integral is regularized by considering it in the complex $n$-dimensional space formally. The extension of the dimension 4 to the complex dimension $n$ is easily done in the Feynman-parametric representation of the Feynman integral. The purpose of my talk is to formulate the theory of complex-dimensional integrals in the general framework and apply it to regularizing singular products in position space. Detailed accounts are presented in my papers $[2,3]$.

The complex $n$-dimensional Minkowski space $M^{n}$ is a product of a one-dimensional Euclidean space $\boldsymbol{R}$ and a complex $(n-1)$-dimensional space $E^{n-1}$ such that the scalar product in $M^{n}$ is defined by the difference between the product in $\boldsymbol{R}$ and the scalar product in $E^{n-1}$. Here $E^{n-1}$ is an abstract vector space equipped with a real-valued, symmetric scalar product. Except for the case in which $n$ is a positive integer, however, $E^{n-1}$ is not a topological space and therefore the number of linearly independent vectors in it is indefinite because it has no complete basis. It is assumed that any finite-dimensional subspace of $E^{n-1}$ is a Euclidean

Received July 14, 1976.

* Research Institute for Mathematical Sciences, Kyoto University, Kyoto 606. 
space. The notion of components $p_{1}, p_{2}, \cdots$ of a vector $p \in E^{n-1}$ is meaningful only with reference to such a subspace. The index $\mu$ of a vector $p_{\mu} \in M^{n}$ takes discrete values only when one works in a finite-dimensional subspace of $M^{n}$.

Let $F\left(p_{\mu}\right)$ be a tempered distribution (or a Fourier hyperfunction) of scalar products $p^{2}, p x^{(1)}, \cdots, p x^{(k)}$, where $p_{\mu}$ is an integration vector and $x_{\mu}{ }^{(1)}, \cdots, x_{\mu}{ }^{(k)}$ are constant vectors of $M^{n}$. Then I define the complexdimensional integral of $F\left(p_{\mu}\right)$ by

$$
\begin{aligned}
\int d^{n} p F\left(p_{\mu}\right) \equiv & \frac{2 \pi^{(n-k-1) / 2}}{\Gamma((n-k-1) / 2)} \int_{-\infty}^{+\infty} d p_{0} \int_{-\infty}^{+\infty} d p_{1} \cdots \int_{-\infty}^{+\infty} d p_{k} \\
& \int_{0}^{\infty} d p_{\perp} p_{\perp}^{n-k-2} F\left(p_{0} ; p_{1}, \cdots, p_{k} ; p_{\perp}\right) .
\end{aligned}
$$

Here $p_{1}, \cdots, p_{k}$ are orthogonal coordinates in a generically $k$-dimensional subspace spanned by the spatial parts $\boldsymbol{x}^{(1)}, \cdots, \boldsymbol{x}^{(k)}$ of $x_{\mu}{ }^{(1)}, \cdots, x_{\mu}{ }^{(k)}$, and

$$
p_{\perp}^{2} \equiv p^{2}-\sum_{j=1}^{k} p_{j}^{2}
$$

If $\boldsymbol{x}^{(1)}, \cdots, \boldsymbol{x}^{(k)}$ happen to be linearly dependent, that is, for example, $F$ is independent of $p_{k}$, then setting $p_{\perp}^{\prime 2}=p_{\perp}^{2}+p_{k}{ }^{2}$, one can easily see that (1) reduces to the expression which is the same as (1) except that $k$ is replaced by $k-1$. Thus the definition (1) does not intrinsically depend on $k$. From this fact it follows that (1) is invariant under a translation of the integration vector $p_{\mu}$, as it should be. Of course, (1) reduces to the ordinary $n$-dimensional multiple integral when $n$ is a positive integer.

The complex-dimensional integral defined by (1) is not manifestly Lorentz invariant, but its Lorentz invariance can be proved. More precisely, (1) can be shown to be a quantity depending only on scalar products formed from $x_{\mu}{ }^{(1)}, \cdots, x_{\mu}{ }^{(k)}$. The proof is carried out by reducing the problem to that for the complex-dimensional Fourier transform ${ }^{1)}$

$$
\begin{aligned}
& \int d^{n} p e^{-i p x} \varphi\left(p^{2}\right)=(2 \pi)^{(n-1) / 2} \int_{-\infty}^{+\infty} d p_{0} \int_{0}^{\infty} d|\boldsymbol{p}||\boldsymbol{p}|^{(n-1) / 2}|\boldsymbol{x}|^{-(n-3) / 2} \\
& J_{(n-3) / 2}(|\boldsymbol{p}||\boldsymbol{x}|) e^{-i p_{0} x_{0}} \varphi\left({p_{0}}^{2}-|\boldsymbol{p}|^{2}\right),
\end{aligned}
$$

where $J_{\nu}$ denotes a Bessel function.

\footnotetext{
1 The right-hand side follows from the polar-coodinate form of (1) with $k=1$.
} 
The complex-dimensional invariant delta functions are defined by

$$
\begin{aligned}
& \Delta_{n}\left(x ; m^{2}\right) \equiv-i(2 \pi)^{-n+1} \int d^{n} p \epsilon\left(p_{0}\right) \delta\left(p^{2}-m^{2}\right) e^{-i p x}, \\
& \Delta^{(1)}\left(x ; m^{2}\right) \equiv(2 \pi)^{-n+1} \int d^{n} p \delta\left(p^{2}-m^{2}\right) e^{-i p x},
\end{aligned}
$$

etc. Their explicit expressions can be calculated by using (3). For example,

$$
\Delta_{n}\left(x ; m^{2}\right)=-\epsilon\left(x_{0}\right) \frac{\left(\sqrt{x^{2}} / m\right)^{(2-n) / 2}}{2^{n / 2} \pi^{(n-2) / 2}} J_{(2-n) / 2}\left(m \sqrt{x^{2}}\right) \theta\left(x^{2}\right)
$$

It is easy to extend the definition of the complex-dimensional integral to the case in which the integrand is a Lorentz-covariant quantity $G_{\mu \ldots \nu,}$, which is defined by

$$
H \equiv y^{\mu} \cdots z^{\nu} G_{\mu \cdots \nu},
$$

where $H$ is a Lorentz-invariant quantity and $y_{\mu}, \cdots, z_{\nu}$ are artificially introduced constant vectors in $M^{n}$. For example, consider $G_{\mu \nu}=p_{\mu} p_{\nu} F\left(p^{2}\right.$, $p x)$. The complex-dimensional integral of $y^{\mu} z^{\nu} G_{\mu \nu}$ is given by (1). Because of the Lorentz invariance of (1) and the proportionality in $y_{\mu}$ and $z_{\nu}$, I can write

$$
\int d^{n} p\left(y^{\mu} p_{\mu}\right)\left(z^{\nu} p_{\nu}\right) F\left(p^{2}, p x\right)=\left(y^{\mu} x_{\mu}\right)\left(z^{\nu} x_{\nu}\right) \Phi_{1}\left(x^{2}\right)+\left(y^{\mu} z_{\mu}\right) \Phi_{2}\left(x^{2}\right),
$$

where $\Phi_{1}$ and $\Phi_{2}$ depend only on $x^{2}$. On introducing an abstract metric tensor $g_{\mu \nu}$ of $M^{n}$, I rewrite (8) as

$$
\int d^{n} p p_{\mu} p_{\nu} F\left(p^{2}, p x\right)=x_{\mu} x_{\nu} \Phi_{1}\left(x^{2}\right)+g_{\mu \nu} \Phi_{2}\left(x^{2}\right) .
$$

Then it can be proved that the formula

$$
g^{\mu \nu} \int d^{n} p p_{\mu} p_{\nu} F\left(p_{\sigma}\right)=\int d^{n} p p^{2} F\left(p_{\sigma}\right)
$$

always holds if and only if one sets $^{2}$

$$
g_{\mu}^{\mu}=n \text {. }
$$

The proof is carried out by showing that to prove (10) is equivalent

${ }^{2}$ Necessity of (11) is well known and is shown easily. 
to proving

$$
\left(g^{\mu \nu} \frac{\partial}{\partial x^{\mu}} \frac{\partial}{\partial x^{\nu}}+m^{2}\right) \Delta_{n}^{(1)}\left(x ; m^{2}\right)=0 .
$$

Finally, I mention the complex-dimensional regularization of singular products in position space. As is well known, the invariant delta functions in the ordinary Minkowski space exhibit light-cone singularities:

$$
\begin{gathered}
\Delta\left(x ; m^{2}\right)=-\frac{\epsilon\left(x_{0}\right)}{2 \pi}\left[\delta\left(x^{2}\right)-\frac{m^{2}}{4} \theta\left(x^{2}\right)+\cdots\right], \\
\Delta^{(1)}\left(x ; m^{2}\right)=-\frac{1}{2 \pi^{2}}\left[\mathrm{P} \frac{1}{x^{2}}-\frac{m^{2}}{4}\left(\log \frac{m^{2}\left|x^{2}\right|}{4}+2 \gamma-1\right)+\cdots\right],
\end{gathered}
$$

where $\mathrm{P}$ and $\gamma$ denote Cauchy's principal value and Euler's constant, respectively. Therefore their products are not well defined. The complex-dimensional extensions $A_{n}$ and $\Delta_{n}^{(1)}$ are, however, continuous on the light cone $x^{2}=0$ if $\operatorname{Re} n<2$. In that region, therefore, any product of $\Delta_{n}$ and $\Delta_{n}^{(1)}$ is always well defined. What I propose is to define singular products in the ordinary Minkowski space by analytically continuing in $n$ the corresponding complex $n$-dimensional products to $n=4$. After lengthy calculations, I have found that the products $\left(\Delta_{n}\right)^{2}, \Delta_{n} \Delta_{n}^{(1)}$, and $\left(\Delta_{n}^{(1)}\right)^{2}$ have no pole at $n=4$. Accordingly, I obtain the regularized expressions for $\Delta^{2}, \Delta \Delta^{(1)}$, and $\left(\Delta^{(1)}\right)^{2}$ unambiguously [2]. They are consistent with another way of definitions

$$
\begin{aligned}
& \Delta\left(x ; m^{2}\right) \Delta^{(1)}\left(x ; m^{2}\right)=2 \epsilon\left(x_{0}\right) \operatorname{Im}\left[\Delta_{F}\left(x ; m^{2}\right)\right]^{2}, \\
& {\left[\Delta^{(1)}\left(x ; m^{2}\right)\right]^{2}-\left[\Delta\left(x ; m^{2}\right)\right]^{2}=4 \operatorname{Re}\left[\Delta_{F}\left(x ; m^{2}\right)\right]^{2}}
\end{aligned}
$$

where $2 \Delta_{F} \equiv i \epsilon\left(x_{0}\right) \Delta+\Delta^{(1)}$ is a boundary value of an analytic function.

\section{References}

[1] Leibbrandt, G., Introduction to the technique of dimensional regularization, Rev. Mod. Phys. 47 (1975), 849-876. Further references are contained therein.

[2] Nakanishi, N., Complex-dimensional invariant delta functions and lightcone singularities, Comm. Math. Phys. 48 (1976), 97-118.

[3] — Lorentz invariance of the complex-dimensional integral, RIMS preprint. 